\title{
Coin Sorter Electrical Control System Design Qixing Liu
}

\author{
Guizhou University School of electrical engineering, automation \\ ZXR67811@163.com, zip code: 550025
}

Keywords: Coin sorter; Electrical control system; Design

\begin{abstract}
Through the coin sorter electrical control system design, to understand the structure of the coin sorter, he mastered it works and use of the main design is simple, accurate sorting, reliable, easy maintenance, low cost advantages. And the system has the potential to expand to meet the future needs of the coin sorter development. Its development prospects, has application value.
\end{abstract}

\section{Introduction}

Coin sorter technology products for the financial industry equipment inventory coin, its main function is to coin counting and sorting coins currency. At present, for supermarkets, public transportation, the vending industry, retail and other industries. Coin sorter looks simple and generous, clear principles of equipment. Simple to understand, affordable, supermarkets, retail and other industries popular. [1]

The biggest advantage of the coin sorter is that it can not only count the number of coins of each denomination will be more clear to separate, and the face of the coins of different countries do not need to adjust the software, only need to adjust the machine's hardware equipment to meet different coin national inventory needs. Therefore, not only occupy a large market share, but also for the cost of production has also been reduced. Research and design of the coin sorter of mechanical and electrical control system is very important practical significance. [2]

\section{Coin Sorter Design}

The Overall Program Design. Coin sorter is designed primarily from the coin's diameter, thickness, separation, count, weight and other aspects to be considered.

Specific separation step is a: First of mixed coins by coin collection funnel, funnel page block credits under rationing by the electric A machine (regular quantitative aims to prevent excessive feeding a coin which led to the separation plate burden too large, causing blockage and separation is not smooth, prevent damage to the motor caused by jammed coins, including coins destruction thickness containing coins of the hole.) portion of mixed credits to the separation disc shown in the Figure, at first we separate denomination is $\$ 1$ coin, so the first stop credits page the distance separating disk design than the $\$ 1$ coin is slightly larger, bigger than the diameter of the other denomination of the coin, as a standard and then after separation experiments produced all the coins can pass, we identified a suitable all kinds of coin diameter. Separation disc is rotated by the motor drive up the use of coins containing coins bore the weight of its own coins isolated on a disc, the disc separated into 45 degree rotation from low to high latch coins. [3]

Each containing coins bore only latch onto a coin containing coins bore the upper end of the separation discs are penetrated currency drain hole, drain hole diameter coin file format is based on a gross (small) a gross (large) fifty cents, one dollar diameter design, since the coins will fall from its own weight so as to fit the diameter of the coin, to achieve separation.

It works. Button switch to start the motor A, B. A high-speed motor is changed by the movement of the low-speed gear, driven gear page flipping coins. B motor driven separation discs, so that the separation disk rotation, the separation plate containing coins latch hole coins, coins from low to high were sent to separate hole, and then the coin size in ascending order from the drain hole credits by gravity, fall, and thus different diameter coins to be separated. [4] 


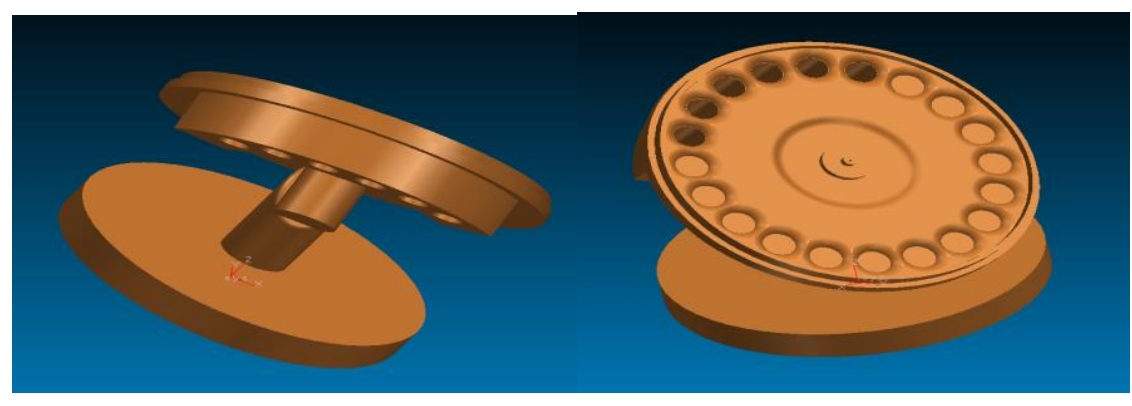

Figure 1. Coin base and the separation discs

The system adopted is the use of light sensors as the basic coin counting sensor; Counting means with a phototransistor as the detection element.

When the coin in succession through the drain holes currency position, the receiver receives the optical signal through an optical signal will receive a will receive $\mathrm{N}$ optical signals through the $\mathrm{N}$, while output at its output electrical pulses $\mathrm{N}$ signal. Use a microcontroller on the falling edge of the output electrical pulse signal is counted, and by LED digital display tubes will count results are displayed. [5]

Control Circuit Design. In order to meet the normal operation of the above system, we can use the programmable controller is designed to be self-developed single-chip printed circuit board. The former short development cycle, strong anti-interference ability, but as mass production, the price is expensive, confidentiality is not good. Ome human and material resources to be put into the development of the latter, only for digital input and output control system, interference relatively easy to solve, as long as the circuit board layout reasonable number, the entire line wiring note of it. and so, The machine uses a hardware circuit design shown in Fig. [6]

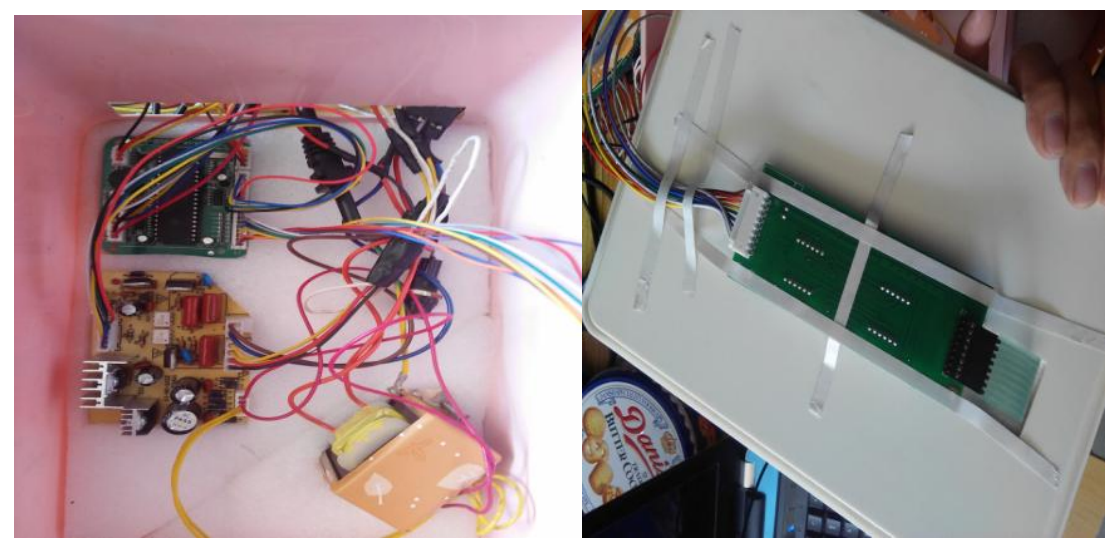

Figure 2. Hardware circuit design

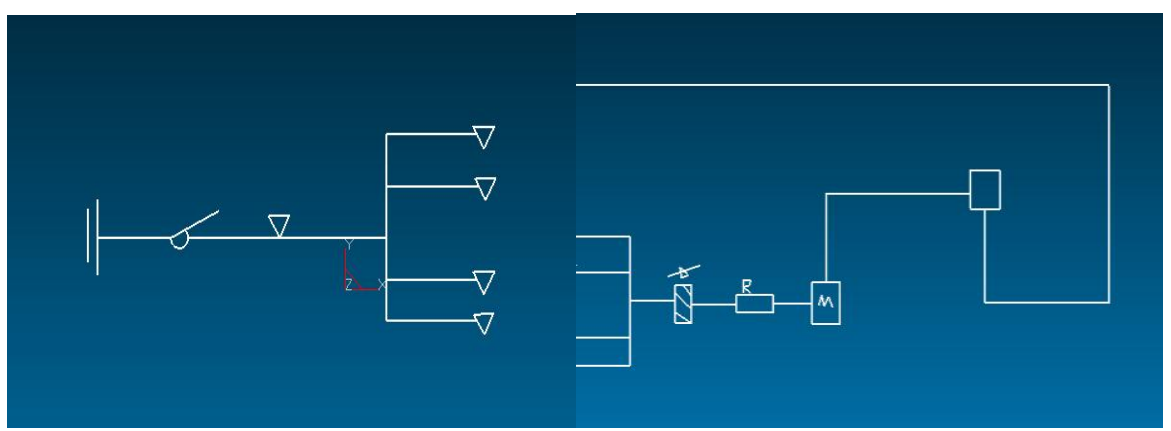

Figure 3. Schematic

\section{Select Motor}

Taking into account the design of the coin sorter machine is intended for retail, banking and 
individual businesses, it should be used in the motor voltage $220 \mathrm{~V}$. Taking into account the loads suffered little, the required power is not large, the use of small power motors. [7]

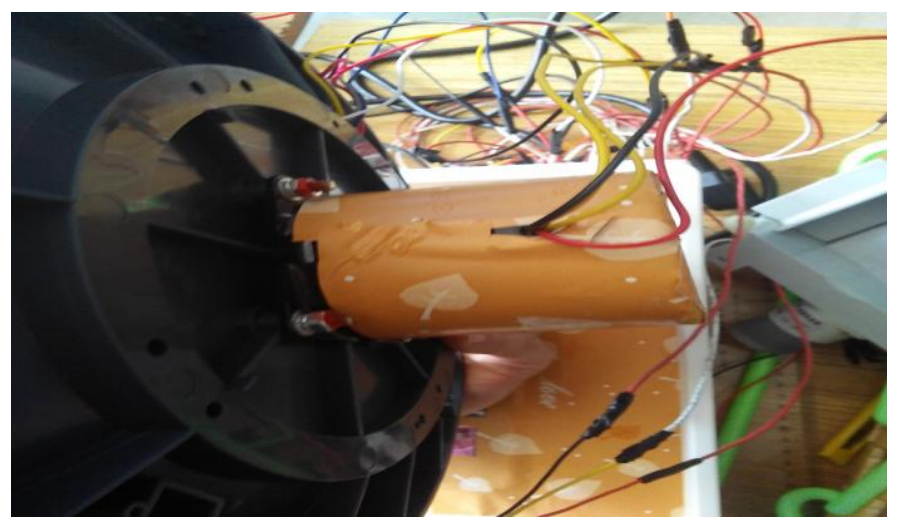

Figure 4. Electric motor

Considering various factors, the choice 60KTYZ series motor.

60TKYZ series motor is a new energy-efficient products, with small size, large capacity, superior starting and operating performance and other characteristics, in line with relevant provisions of international standards IEC and to achieve the same frame size single, three-phase asynchronous motors the same level, to improve the single , three-phase motor interchangeability and versatility, are widely used in refrigeration machines, pumps, fans, small machine tools as well as sales of agricultural and household appliances and so on. The main parameters of the motor: Model: $60 \mathrm{TKYZ}$, voltage: $220 \mathrm{~V}$, power: $0.45 \mathrm{KW}$, synchronous speed: $15 \mathrm{r} / \mathrm{min}$, frequency: $50 \mathrm{HZ}$, efficiency: $68 \%$. [8]

Energy devices is the electrical energy into mechanical energy, which normally is a common motor.

Execution means is converted into electrical energy to mechanical energy of the moving means coin sorter as motor AB. The control device is a power filter, control, and change means the coin sorter and coil voltage fuse, different combinations of these original composition of the work to complete the clearing system. [9]

Other auxiliary device means a device other than these three, such as the protection plate, support base, as well as other body care. They work to ensure that the coin sorter system reliable and stable work have a significant role. Transmission medium energy transfer medium, namely circuit, circuit guides. [10]

\section{Conclusion}

The machine according to the size of the diameter of the coin denominations were clearing method, although does not have the function of pseudo-debate, but it can quickly and accurately according to China's current coin denominations were clear points. The main feature of the design is simple, accurate sorting, reliable, easy maintenance and low cost. And the system has the potential to expand to meet the future needs of the coin sorter development. In banks, transport companies and other departments, greatly improve their work efficiency and reduce costs. Therefore, this product has a high application value, and its development prospect is very broad.

\section{References}

[1] Xudong Deng: Research and development of electrical control system for distributed control system [J], Hubei electric power system, Vol. 25 No.5, p.21.

[2] Shenghua Feng: A discussion on the development of electrical control of elevator [J]. science and technology information, Vol. 21 (2009) 
[3] Liling Liu: The development technology of electrical control technology [J]. Journal of Beijing Electric Power College, (2011) No.3, p.31.

[4] Guowei Zhu and Guoxiu Wang: Graphic LCD Module MGLS-19264. Application of Intelligent geophone [J]. Beijing Polytechnic College, (2003:2)

[5] Changchu Liao: PLC programming and application (Mechanical Industry Press, China 2014)

[6] Kewang Pang and Wenhua Yuan: PLC electrical control system design and application (China Electric Power Press, China 2014)

[7] Zhiyong Jia, Xueyong Li and Gao Hongyan: introduction to the application of PLC (China Electric Power Press, China 2014)

[8] Huafeng Mei: electrical equipment and control (China Water Conservancy and Hydropower Press, China 2015)

[9] Pin Wan and Dejie Lin: Electrical Testing Technology (Mechanical Industry Press, China 2015)

[10] Songwei Huang and Jinhui Zou: electrical control and PLC application technology (Electronic Industry Press, China 2015) 